\title{
Breve revisão sobre os estudos realizados com radiotraçadores artificiais para a obtenção de parâmetros físico-químicos em matrizes ambientais na Floresta Experimental de Itacuruçá/RJ
}

\section{Alfredo Victor Bellido Bernedo ${ }^{1 *}$ \\ Edimar Carvalho Machado² \\ Fátima de Paiva Canesin ${ }^{1}$ \\ Katia Noriko Suzuki ${ }^{3}$ \\ Luis Fernando Bellido Bernedo ${ }^{4}$ \\ ${ }^{1}$ Departamento de Físico-Química, Instituto de Química, Universidade Federal Fluminense (UFF) - Niterói (RJ), Brasil.}

${ }^{2}$ Instituto Federal de Educação, Ciência e Tecnologia do Rio de Janeiro - Nilópolis (RJ), Brasil.

${ }^{3}$ Laboratório de Instrumentação Nuclear (LIN-COPPE), Universidade Federal do Rio de Janeiro (UFRJ) - Rio de Janeiro (RJ), Brasil.

${ }^{4}$ Instituto de Radioproteção e Dosimetria (IRD/CNEN) - Rio de Janeiro (RJ), Brasil.

*Autor correspondente: alfredobellido@gmail.com

\section{Resumo}

O Laboratório de Química Nuclear e Radioquímica da Universidade Federal Fluminense (Niterói/RJ), desde 1994, desenvolve projetos de pesquisa na área ambiental junto ao programa de Pós-graduação em Geoquímica Ambiental, onde utiliza a técnica com radiotraçadores para o entendimento de processos em diferentes compartimentos geoquímicos principalmente em áreas de manguezais. O presente trabalho mostra, por meio de estudos-caso, a aplicação da técnica de radiotraçadores artificiais na Floresta Experimental de Itacuruçá/RJ para estudos de cinética e especiação de elementos químicos em ecossistemas dinâmicos de manguezal. O radiotraçador Manganês-54 foi usado para a compreensão dos processos cinéticos da oxidação do manganês, na coluna d'água com obtenção das constantes específicas da reação de oxidação. No caso do Iodo-123 e Iodo-131, os estudos demonstraram o potencial da utilização de radiotraçadores no entendimento do comportamento geoquímico do iodo na coluna d'água. No último caso, foram apresentados os radiotraçadores como uma ferramenta na análise "não destrutiva" de matrizes ambientais que podem auxiliar na elucidação da interação da bioturbação e da rizosfera entre os diferentes compartimentos geoquímicos que compõem o complexo ambiente de manguezal. Palavras-chave: cinética, manguezal, radiotraçadores, sedimentos.

\section{Abstract}

The Radiochemistry and Nuclear Chemistry Laboratory located in the Universidade Federal Fluminense (Niterói/RJ), since 1994, develops research projects in the Post-graduate program in Environmental Geochemistry, which uses the technique with radiotracers for understanding geochemical processes in different compartments mainly in mangrove areas. This literature review of data already published by the Laboratory shows for kinetic studies and speciation of chemical elements in the dynamic ecosystems of mangrove swamp by means of a studies-case application of the artificial radiotracers technique in the Experimental Forest of Itacuruçal/RJ. The radiotracer manganese-54 was used for the understanding of kinetic processes of oxidation of manganese in the water column with obtaining specific constant of the reaction of oxidation. In the case of iodine-123 and iodine-131, these studies have demonstrated the potential of the use of radiotracers in the understanding of the geochemical behavior of iodine in the water column. In the latter case were presented the radiotracers as a tool in the analysis "non-destructive" of environmental matrices, which may help to elucidate of the interaction of bioturbation and rhizosphere between different compartments geochemical that compose the complex environment of mangrove. Keywords: kinetics, mangrove, radiotracers, sediment. 


\section{INTRODUÇÃO}

A radiação de ocorrência natural é proveniente de três fontes: a radiação cósmica (que tem origem no sol e no espaço extraterrestre), radiação cosmogênica (resultado do bombardeamento constante da atmosfera superior pelos raios cósmicos, por exemplo, Carbono-14, Sódio-22) e a radiação dos elementos radioativos existentes na crosta terrestre (elementos radioativos naturais, como as famílias radioativas do Urânio-238, Urânio-235 e do Tório-32). Os radiotraçadores artificiais são obtidos pelo homem mediante o bombardeio de núcleos atômicos com partículas de energia elevada, especialmente nêutrons, utilizando-se reatores nucleares e também de prótons, partículas $\alpha$, ou outros íons mais pesados, utilizando-se dos aceleradores. Esses radioisótopos são empregados com diferentes finalidades como na medicina (Tecnécio-99m e Iodo-131, que são utilizados para diagnóstico e terapia de câncer), na área alimentícia (conservação de alimentos pela irradiação de raios $\gamma$ por fonte de Cobalto-60), na área industrial (aplicação da técnica da gamagrafia, utilizando a radiação gama para a verificação de defeitos em válvulas, asas e turbinas de avião), entre outras (Bellido \& Latini 2010).

Traçador é um elemento, espécie química ou partícula que por suas características físico-químicas permite seguir o comportamento do mesmo dentro de um determinado sistema que se deseja estudar. Quando é utilizado um isótopo radioativo, chamamos esse elemento de radiotraçador ou traçador radioativo. $\mathrm{O}$ radioisótopo é um nuclídeo radioativo do elemento químico, portanto, possui as mesmas propriedades químicas e físicas do elemento natural. A única diferença é a sua propriedade em emitir radiação durante seu processo de desintegração até atingir o estado fundamental estável de um elemento químico. Esta radiação pode ser medida por um detector que consiste, em linhas gerais, de um elemento ou material sensível à radiação e de um sistema, na maioria das vezes eletrônico, que registra o resultado da interação, expressando-o em termos de uma grandeza de medição [atividade $=$ becquerel $(\mathrm{Bq})]$ dessa radiação que interagiu com o elemento ou meio empregado (Silva et al. 2009).

Em termos gerais, as vantagens da utilização de radiotraçadores em estudos com matrizes ambientais são baseadas em:

(a) as propriedades da radiação dos traçadores geralmente não são afetadas pelas condições clássicas físicas e químicas da amostra, tais como a temperatura, pressão, ou presença e concentração de outros elementos químicos;

(b) a radiação emitida é facilmente detectada e determinada em detectores adequados com alta precisão e sensibilidade (em torno de $10^{-16}$ a $10^{-6} \mathrm{~g}$ );

(c) o radiotraçador pode ser obtido comercialmente com atividades desejadas e alto grau de pureza radionuclídica e radioquímica;

(d) o acompanhamento do seu comportamento é feito através da radiação emitida não sendo afetado pela complexa matriz da amostra, eliminando a necessidade de gerar curvas de calibração; e

(e) a quantidade adicionada é muito pequena, não alterando, assim, a concentração natural da espécie estudada.
As desvantagens são na maior parte relacionadas com a área operacional tais como: a observância da licença do seu uso e medidas de radioproteção adequadas nos laboratórios de pesquisa e com os pesquisadores, bem como o alto custo pela necessidade de importação ou pela restrição em face ao número escasso de locais de produção (Ambe et al. 2003; Chopin et al. 1995).

Apesar das dificuldades apresentadas no seu emprego, Croot et al. (2011) destacaram o papel da técnica analítica utilizando os radiotraçadores em relação às técnicas convencionais de análise. Ressaltaram também o fato de os radiotraçadores terem sido imprescindíveis para o desenvolvimento de várias descobertas importantes em Química e Oceanografia Biológica, tais como: as medições de produtividade primária com Carbono-14, utilização de radioisótopos de ferro e cádmio para estudos básicos em Biologia Marinha, a quantificação da cinética de troca entre diferentes espécies de metal em solução e o desenvolvimento de modelos para a cinética de absorção de metais pelo fitoplâncton.

Payne et al. (2004) citaram como vantagens do uso de traçadores radioativos no acompanhamento de processos ambientais em microcosmo (laboratório), em estudos de caso na Austrália: a grande gama de elementos que podem ser estudados; realização de experimentos multielementares (estudos simultâneos de vários radioisótopos); e viabilidade de estudo de mecanismos, reversibilidade e cinética de reações ambientais em réplicas com poucas alterações do local de origem da amostra.

No estudo da cinética de uma reação, é necessário acompanhar a variação da concentração das espécies envolvidas durante o tempo. As interações que as espécies em estudo sofrem com os outros componentes presentes na amostra são complexas e podem influenciar na especiação do elemento em estudo. O acompanhamento do processo envolvido através de métodos convencionais que requeiram tratamento prévio para a eliminação dos possíveis interferentes da matriz pode inviabilizar o acompanhamento cinético ou levar a interpretações equivocadas. A opção pela técnica de radiotraçadores tem se revelado bastante eficaz, pois permite acompanhar a radiação emitida pelo radiotraçador na complexa matriz da água e do sedimento.

Nas últimas décadas, na Floresta de Itacuruçá localizada na Baía de Sepetiba, estado do Rio de Janeiro, observa-se uma considerável expansão industrial e urbana na região que tem resultado em uma série de impactos ambientais devido ao progressivo aumento no lançamento de efluentes domésticos e industriais, os quais são ricos em metais e outras substâncias potencialmente tóxicas. Um dos maiores passivos ambientais do estado do Rio de Janeiro fica no município de Itaguaí, no terreno onde funcionou a Companhia Mercantil e Industrial Ingá, produtora de zinco e sulfato de zinco. Após a falência da companhia em 1998, a área foi abandonada com grande quantidade de água contaminada com metais pesados, formando uma "bacia" de $260 \mathrm{mil} \mathrm{m}^{2}$. Parte vazou, afetando as águas da baía de Sepetiba. O vazamento expôs 
o risco permanente em causar desastre ecológico na região de mangues da baía de Sepetiba, antigo reduto da pesca artesanal fluminense (Cortez 2006).

No momento, há diversos empreendimentos industriais nessa região, dentre os quais a construção na ilha da Madeira, região próxima à área de pesquisa deste trabalho, de um estaleiro e base naval para a construção de submarinos convencionais e de propulsão nuclear (Arthou 2011). Isso aumenta o interesse na área por coleta de dados de monitoração de radionuclídeos e também de estudos envolvendo radiotraçadores que poderão vir a fornecer modelos para o entendimento de comportamento do ecossistema da Baía de Sepetiba, podendo ser utilizados no futuro para fins comparativos na análise de impactos ambientais após o início da operacionalização daquele estaleiro.

O Laboratório de Química Nuclear e Radioquímica, do Departamento de Físico-Química, localizado no Instituto de

\section{MATERIAIS E MÉTODOS}

A metodologia foi compartilhada de forma geral nos três estudos apresentados quanto à aplicação dos radiotraçadores e na área de estudo.

\section{1. Área de Estudo}

Próximo à cidade de Itacuruçá localizada na Baía de Sepetiba (Figura 1), no lado norte do canal de Itacuruçá, numa área de aproximadamente 13 hectares, está localizada uma floresta de manguezal constituída principalmente pelas espécies Rhizophora mangue L.,
Química da Universidade Federal Fluminense (UFF/RJ), desde 1990 desenvolve atividades na área didática e de pesquisa. A partir de 1994 têm sido desenvolvidos projetos de pesquisa junto ao programa de pós-graduação em Geoquímica Ambiental da UFF/RJ, dentre eles, na linha de pesquisa "Estudo de medidas de parâmetros físico-químicos utilizando radiotraçadores naturais e artificiais em matrizes ambientais" com estudos de determinação de parâmetros físico-químicos para entendimento de processos em diferentes compartimentos geoquímicos, utilizando-se radiotraçadores produzidos por aceleradores ou reator nuclear e/ou radiotraçadores naturais. O objetivo deste trabalho é divulgar por meio de uma breve revisão, os resultados e discussão de dados já publicados, dos estudos da interface água-sedimento da Floresta de Itacuruçá/RJ, demonstrando o potencial dos radiotraçadores artificiais em áreas multidisciplinares da Geoquímica Ambiental.
Avicennia schauriana e Laguncularia racemosa. O solo da floresta é argilo-arenoso na parte baixa e areno-argiloso na parte alta do mangue. O local de coleta das amostras se encontra adjacente ao canal da maré na região do mangue baixo.

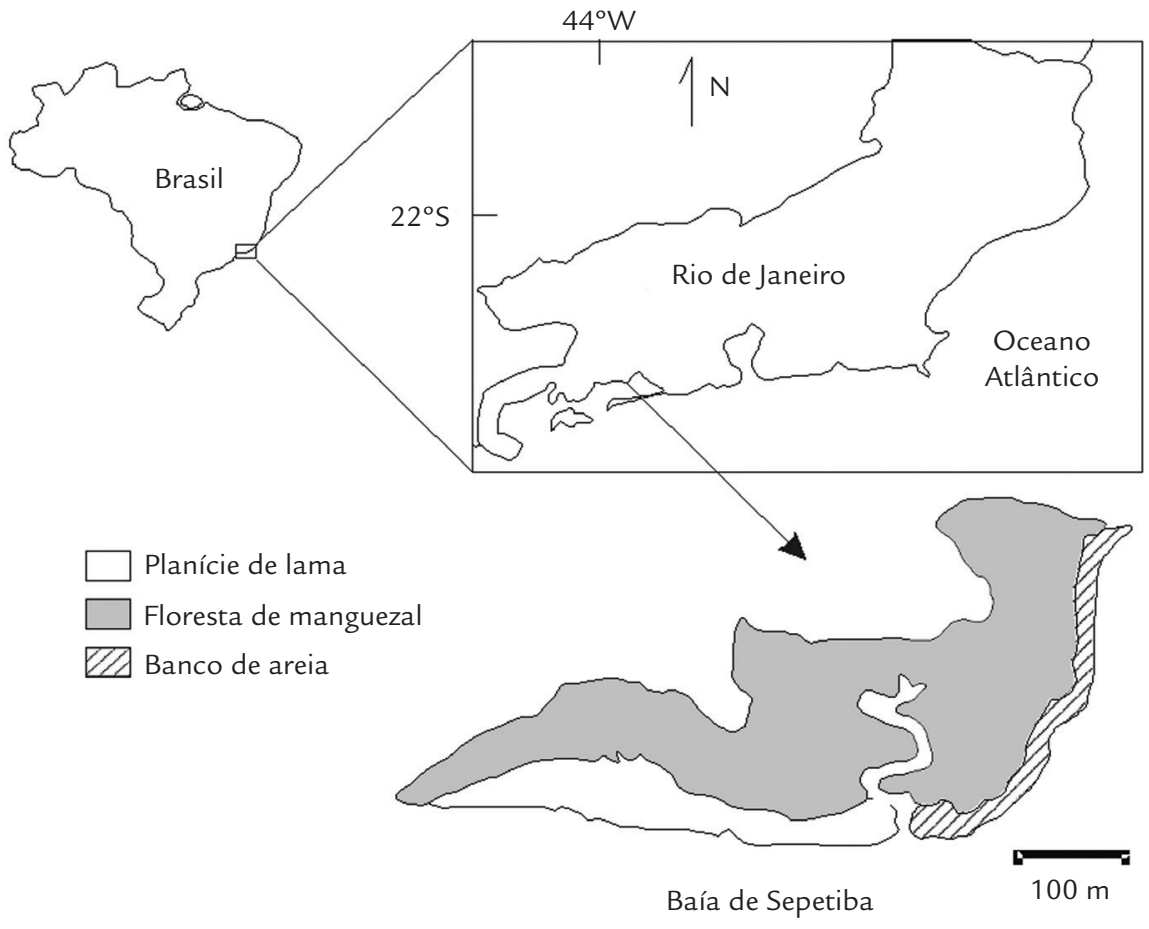

Figura 1

Mapa com localização da área de estudo na baía de Sepetiba. 


\subsection{Radiotraçadores}

Os radiotraçadores artificiais (Tabela 1) utilizados neste trabalho foram obtidos em convênio com o Instituto de Pesquisas Energéticas e Nucleares (IPEN-CNEN/SP) pela irradiação de alvos padrões no Reator Nuclear de Pesquisa (IEA-R1).

As medidas radionuclídicas foram feitas usando a espectroscopia de radiação gama do Laboratório de Radioquímica (IQ/UFF) constituída de um detector de germânio hiperpuro (HPGe) e eletrônica associada. $\mathrm{Na}$ espectroscopia gama, a caracterização dos radiotraçadores foi determinada pela quan- tificação dos fotopicos com energias específicas (Tabela 1). A eficiência do detector em função das energias gama foi determinada pelas contagens de fontes calibradas e a aquisição dos espectros realizada com a ajuda do software Maestro for Windows versão 5.10, EG\&G ORTEC, com análise das áreas dos espectros por meio do software Aptec versão 3.95 da Aptec Instruments Ltda. Nas medidas das amostras, procurou-se manter a mesma geometria detector-fonte e o erro propagado foi inferior a $5 \%$.

\begin{tabular}{|c|c|c|c|}
\hline & & & \\
\hline \multirow{6}{*}{$\begin{array}{r}\text { Tabela } 1 \\
\text { Características dos radiotraçadores } \\
\text { utilizados nos experimentos (Dela- } \\
\text { croix et al. 2002). }\end{array}$} & Radiotraçador & Meia-vida & Método de detecção (keV) \\
\hline & ${ }^{51} \mathrm{Cr}$ & $27,7 \mathrm{~d}$ & $\gamma(320)$ \\
\hline & ${ }^{54} \mathrm{Mn}$ & $312,2 \mathrm{~d}$ & $\gamma(836)$ \\
\hline & ${ }^{75} \mathrm{Se}$ & $119,8 \mathrm{~d}$ & $\gamma(136)$ \\
\hline & ${ }^{123} \mid$ & $13,13 \mathrm{~h}$ & $\gamma(159)$ \\
\hline & ${ }^{131} \mathrm{I}$ & $8,04 \mathrm{~d}$ & $\gamma(367)$ \\
\hline
\end{tabular}

\subsection{Comportamentos Cinéticos do Manganês}

Para o acompanhamento cinético, amostras de $250 \mathrm{~mL}$ de água foram coletadas em diferentes pontos do mangue e em diferentes alturas de maré para verificar a influência das marés vazantes e enchentes na cinética de oxidação, pois a hidrologia, hidrodinâmica e os parâmetros físico-químicos sofrem influencias diárias pelos ciclos de maré. A metodologia para a incubação foi modificada de Sunda e Huntsman (1987). As amostras de água de 11 campanhas foram incubadas no laboratório com o radiotraçador, com tomada do tempo inicial. Após tempos variados de incubação foram filtradas alíquotas de $30 \mathrm{~mL}$, a vácuo, com filtros Millipore de 0,22 $\mu \mathrm{m}$, em intervalos de tempo definidos. Os filtros foram lavados com água do mangue, armazenados em placas de Petri e depois de secos foram contados por 600 segundos no detector de germânio hiperpuro. Os filtrados também foram contados nas mesmas condições. Para verificar os efeitos de superfície, ou efeito abiótico na cinética de oxidação foi adicionado, antes da incubação com o Mn-54, formol a 3\% para eliminar possíveis efeitos da ação de microrganismos como catalisadores da cinética de oxidação do Mn. Como resultado da contagem, observou-se que as atividades dos filtrados diminuíram com o tempo enquanto a atividade dos filtros aumentou indicando a precipitação dos óxidos de $\mathrm{Mn}$. As atividades nos filtros e filtrados determinadas variaram de 3 a $3000 \mathrm{~Bq}$.

\subsection{Estudo do Equilíbrio Químico do lodo na Água do Mar}

Em virtude da necessidade de se incubar o iodo na forma de iodato como espécie química, essa espécie foi obtida a partir da oxidação de iodeto radioativo de acordo com Palágyi et al. (1971). A separação de Iodeto e Iodato nas amostras para análise radioativa foi feita através de cromatografia em coluna suportada por alumina (Machado et al. 2001).

Amostras de água foram coletadas em frascos de polietileno com capacidade de 1 litro e levadas acondicionadas ao laboratório. Três litros foram filtrados utilizando membranas de $0,22 \mathrm{~mm}$ para a retenção dos micro-organismos, enquanto outros 3 litros não foram filtrados. Os volumes filtrado e não filtrado foram divididos em duas metades, tendo sido adicionado iodato-131 em uma delas e iodeto-131 na outra. Em seguida, as amostras incubadas foram acondicionadas em frascos de $500 \mathrm{~mL}$ cada. Alíquotas de $20 \mathrm{~mL}$ eram retiradas, filtradas em membrana de $0,45 \mathrm{~mm}$ e separadas em coluna cromatográfica. Os eluentes e o filtro obtidos foram levados ao detector de germânio para quantificar a sua especiação.

\subsection{Estudos de Parâmetros Físico-Químicos na Interface Água-Sedimento}

O estudo da remoção dos radiotraçadores da coluna d'água para o sedimento e sua posterior difusão em ambiente óxico foi feito coletando-se três testemunhos $(0-8 \mathrm{~cm})$ utilizando-se tubos de Plexiglas $(4,4 \mathrm{~cm}$ de diâmetro e $25 \mathrm{~cm}$ de comprimento) com espaçamento de $10 \mathrm{~cm}$ entre eles. Após a introdução do tubo no sedimento, a parte inferior do tubo foi tampada com uma rolha de borracha coberta por um filme de PVC e a parte superior com plástico. Os tes- temunhos (denominados A, B e C) para preservar o estado original das amostras, foram transportados imediatamente até o laboratório, mantendo-se uma coluna d'água de $5 \mathrm{~cm}$ acima dos sedimentos. Esta água foi trocada por $150 \mathrm{~mL}$ de água incubada com um coquetel de radiotraçadores com atividades médias iniciais $( \pm \mathrm{DP})$ de $22,0 \pm 0,6,10,7 \pm 1,1 \mathrm{e}$ $65,2 \pm 1,0 \mathrm{~Bq} / \mathrm{mL}$ para Selênio-75, Cromo-51 e Cobalto-60, respectivamente. 
A coluna d'água foi constantemente aerada, purgando-se ar. O tempo da amostragem foi de 0,5 a $115,0 \mathrm{~h}$ totalizando 10 amostras retiradas e com suas respectivas atividades medidas. No final do experimento, a coluna d'água foi removida, o sedimento (total de $8 \mathrm{~cm}$ ) foi fatiado em fatias de $1 \mathrm{~cm}$ cada e a

\section{RESULTADOS E DISCUSSÃO}

\subsection{Comportamento Cinético do Manganês}

No ambiente de manguezal ocorrem mudanças físico-químicas e processos biogeoquímicos que são influenciados pelas variações das amplitudes de marés. Essa dinâmica permite a troca de materiais particulados e dissolvidos entre o mangue e as águas estuarinas adjacentes com grande efeito na produtividade secundária destas águas. O material particulado presente na água, associado à matéria orgânica, entra no mangue juntamente com óxidos hidratados de ferro e manganês. Estes são responsáveis pela sorção de metais como zinco, cádmio e outros, principalmente de valência $2^{++}$. Nos ambientes redutores, como o mangue, os hidróxidos ácidos são reduzidos liberando os metais pesados, que juntamente com o ferro tendem a formar sulfetos. O manganês não forma sulfeto estável nas condições do mangue permanecendo na coluna d'água nas formas dissolvidas e particulada. A ciclagem redox do manganês possui um papel importante como catalisadora da oxidação da matéria orgânica pelo oxigênio. As reações de oxidação do manganês com o oxigênio molecular são descritas com três etapas num mecanismo heterogêneo:

$$
\begin{aligned}
& \mathrm{Mn}(\mathrm{II})+1 / 2 \mathrm{O}_{2}+\mathrm{H}^{2} \mathrm{O} \rightarrow \mathrm{MnO}_{2}(\mathrm{~s})+2 \mathrm{H}^{+} \text {(lenta) } \\
& \mathrm{Mn}(\mathrm{II})+\mathrm{MnO}_{2}(\mathrm{~s}) \rightarrow \mathrm{Mn}(\mathrm{II}) \mathrm{MnO}_{2}(\mathrm{~s}) \text { (rápida) } \\
& \mathrm{Mn}(\mathrm{II}) \mathrm{MnO}^{2}(\mathrm{~s})+1 / 2 \mathrm{O}_{2} \rightarrow 2 \mathrm{MnO}_{2}(\mathrm{~s}) \text { (lenta) } \\
& -\mathrm{d}[\mathrm{Mn}(\mathrm{II})] / \mathrm{dt}=\mathrm{k}_{1}{ }_{1}[\mathrm{Mn}(\mathrm{II})]+\mathrm{k}_{2},[\mathrm{Mn}(\mathrm{II}) \mathrm{MnOx}],
\end{aligned}
$$

onde as constantes específicas de velocidade são de pseudo-ordem, em relação ao oxigênio e a hidroxila.

O estabelecimento de uma metodologia capaz de entender como a cinética de oxidação do manganês se desenvolve em ambientes dinâmicos, como um ecossistema de manguezal, fica facilitado com a incubação com o radiotraçador atividade das fatias medida no detector de HPGe. Os inventários das atividades dos radionuclídeos foram calculados para cada fatia de sedimento como produto da atividade (Bq/g) e a densidade do sedimento $\left(\mathrm{g}_{\mathrm{cm}} \mathrm{cm}^{-3}\right.$. Essa densidade foi obtida por meio da determinação da massa seca do sedimento a $50^{\circ} \mathrm{C} \mathrm{em} 72 \mathrm{~h}$. artificial Manganês-54. Estudos de Morgan \& Stumm (1965) sugeriram uma equação cinética para a oxidação do manganês, em águas naturais. No modelo proposto, a cinética é autocatalítica com um primeiro termo da equação cinética representando um mecanismo homogêneo e uma constante de velocidade $\mathrm{k}^{\prime 1}$ de pseudo-primeira ordem, dependente da concentração do Mn (II) e um segundo termo, representando um mecanismo heterogêneo devido à oxidação na superfície do óxido de manganês, também apresentando uma constante de velocidade de pseudo-primeira ordem, $\mathrm{k}^{\prime 2}$ dependente das concentrações do $\mathrm{Mn}$ (II) e do $\mathrm{MnOx}$. Efeitos como quantidade de material particulado, aumento da área superficial, temperatura, teor de oxigênio dissolvido e a presença de bactérias aumentam a taxa de oxidação. As bactérias influenciam a cinética com a função de catalisadoras na ciclagem do manganês aproveitando a energia liberada na oxidação para o crescimento quimiolitotrófico.

As regressões lineares em diagramas de Ln A x tempo para as primeiras 16 horas de incubação, apresentaram um comportamento linear sugerindo uma cinética de primeira ordem para o início da oxidação e determinação da constante k'1 que variou de $1,0 \times 10^{-5}$ a 4,0x10-5s (Figura 2). O comportamento variou de modo não linear após 16 horas em média, sugerindo um possível mecanismo heterogêneo e autocatalítico, como também verificado por Morgan (1967).

A influência das bactérias na oxidação do manganês foi evidenciada com diminuição das constantes de velocidade. Variações nos parâmetros $\mathrm{pH}$, salinidade, oxigênio dissolvido e temperatura afetaram as constantes de velocidade, em especial a cinética homogênea, sugerindo que a variação está associada a efeitos de troca de água entre o mangue e a água da Baía de Sepetiba. Embora a cinética heterogênea tenha sido evidenciada após um período de maré, ainda não se determinou se os óxidos formados precipitam no mangue ou são exportados para áreas adjacentes da Baía de Sepetiba (Canesin 2000).

\subsection{Estudo do Equilíbrio Químico do lodo na Água do Mar}

A geoquímica do iodo na água do mar tem se mostrado bastante complexa e intrigante. Estudos têm mencionado a dificuldade em se interpretar os resultados obtidos para esse elemento. Em parte, devido às baixas concentrações de suas espécies na água do mar. O iodo está presente na água do mar, principalmente, nas formas iodeto e iodato. A concentração total do iodo, em água do mar, é aproximadamente 0,5 mM (Wong \& Hung, 2001).
Sillén (1961) mostrou que para a reação de equilíbrio:

$$
\mathrm{IO}^{3-}+6 \mathrm{H}^{+}+6 \mathrm{e}^{-} \leftrightarrow \mathrm{I}^{-}+3 \mathrm{H}^{2} \mathrm{O}(\mathrm{pK}=110)
$$

nas condições do oceano (pE 12,5 e pH 8,1), a razão $\overline{\text { [iodato] }}$ é igual a $10^{-13,5}$ 
Portanto, concentração de iodeto em água do mar deveria ser muito menor do que a de iodato. Porém, isso não é observado, pois o iodeto é encontrado em águas superficiais em concentração maior do que o esperado, podendo até chegar a $50 \%$ do iodo total (Wong 1995, Waite \& Truesdale 2003). A elevada concentração de iodeto em água superficial tem indicado a participação do iodo em processos mediados por micro-organismos (Rebello et al. 1990, Edwards \& Truesdale 1997). Por isso, há grande interesse em se entender os processos que mantêm este desequilíbrio na água do mar. Nesse contexto, busca-se com este estudo comparar a interconversão entre iodeto e iodato através do iodo-131 em amostras contendo atividade biológica e amostras com atividade biológica reduzida.

Ao observar os resultados, na Figura 3, nota-se que a transformação de iodeto em iodato ocorre de maneira similar entre as amostras filtradas e não filtradas, indicando assim, que a participação biológica não demonstrou possuir papel fundamental no processo de oxidação do iodeto a iodato. Contudo, é interessante notar que há uma pequena associação do iodo no material particulado retido no filtro. Brandão e colaboradores (1994) observaram incorporação de iodeto pelos zooplâncton e/ou fitoplâncton durante seus experimentos. A oxidação de iodeto em iodato é um processo extremamente lento nas condições encontradas na água do mar, podendo levar a um tempo de meia vida de dezenas de anos e até mesmo não ser observada em vários dias de incubação e na presença de oxigênio (Farrenkopf et al. 1997). Entretanto, nossos resultados mostraram que ocorreu até $20 \%$ de oxidação do iodeto adicionado nos primeiros 3 dias de experimento.

Diferentemente do comportamento observado entre as amostras incubadas com iodeto-131, observou-se significativa diferença entre a formação de iodeto nas amostras sem filtrar e nas filtradas a partir da incubação com iodato-131, demonstrando assim, significativa alteração na conversão de iodato em iodeto em amostras sem filtração.

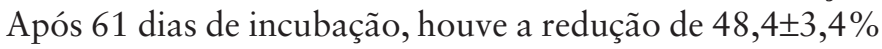
do iodato-131 adicionados nas amostras não filtradas para iodeto-131, enquanto nas amostras que sofreram processo
Figura 2 Cinética do Manganês.

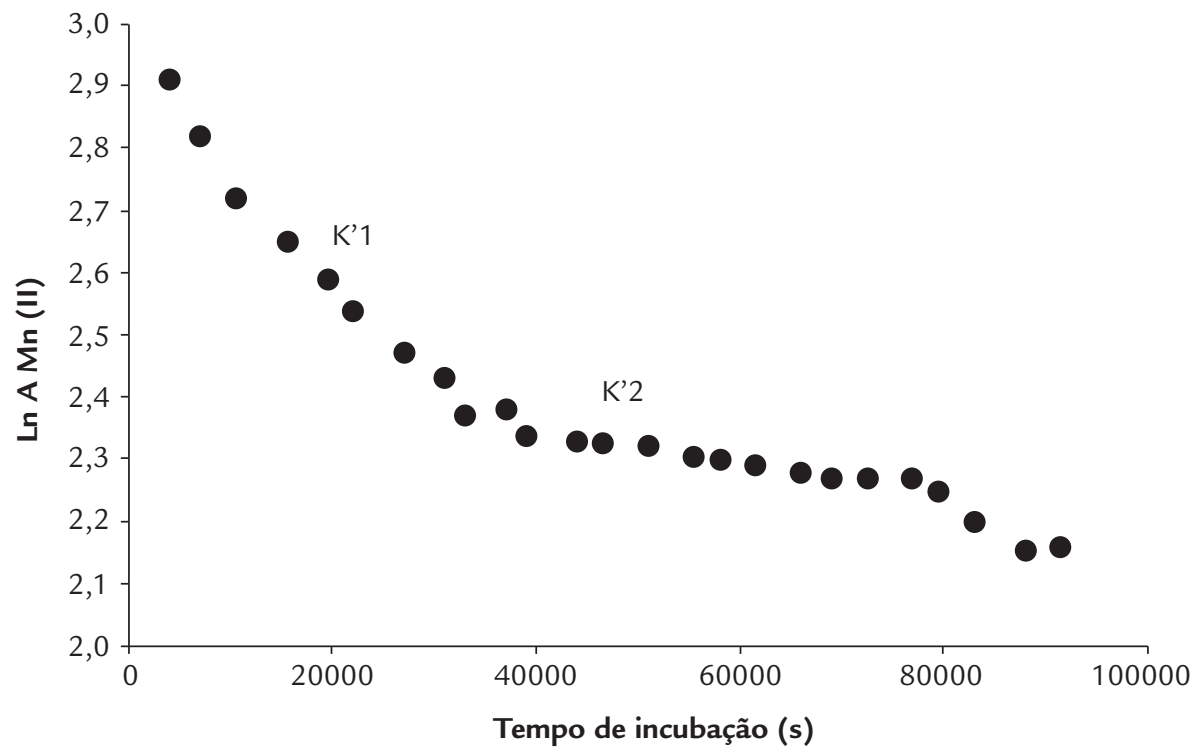

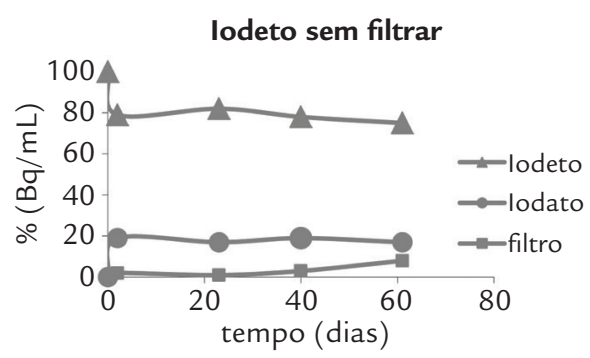

lodeto sem filtrar

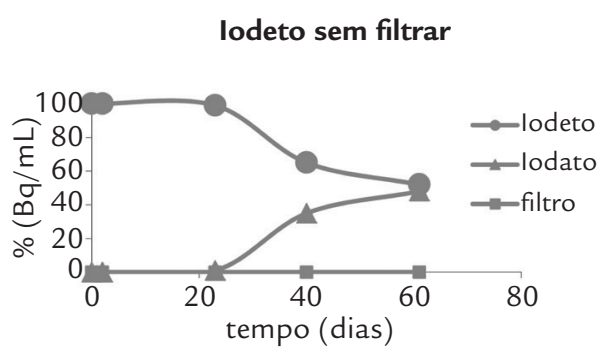

lodeto filtrado
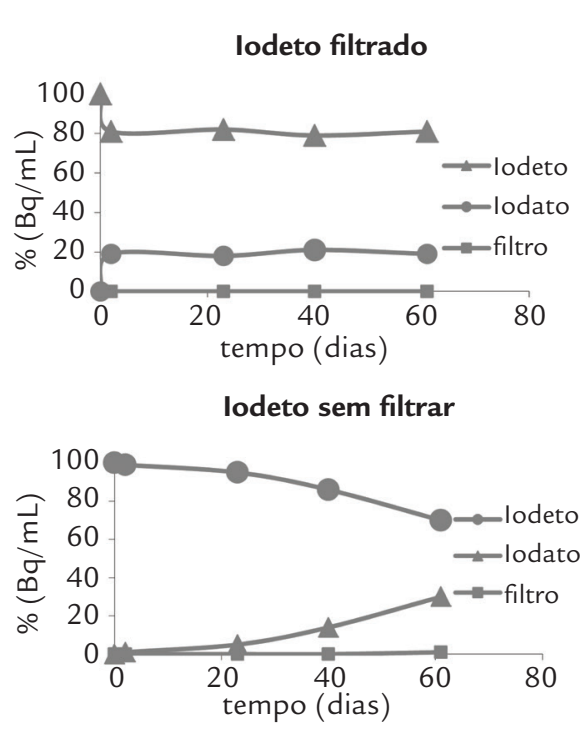

Porcentagem das atividades de iodo131 em amostra filtrada e não filtrada. 
de filtração, houve a formação de $19,8 \pm 10,0 \%$ de iodeto durante o processo de redução do iodato-131. Esses resultados indicam que a atividade biológica contribui significativamente na formação de iodeto a partir do iodato e, mesmo em amostras com menor atividade biológica, permite a redução do iodato a iodeto, confirmando assim, que há um desequilíbrio que faz com que o iodeto esteja em concentração equivalente ao iodato na água do mar, que seria a espécie termodinamicamente favorecida.

É interessante notar que os processos responsáveis pela redução do iodato nas amostras de água ocorrem em um tempo bastante diferente daqueles responsáveis pela oxidação do iodeto, visto que apenas uma pequena parte do iodeto é formada nos primeiros três dias de incubação, período no qual toda a oxidação do iodeto foi observada nas amostras incubadas com iodato. Mesmo durante os 23 dias de incubação, o iodato-131 nas amostras filtradas sofreu conversão em torno de $5 \%$ de iodato para iodeto. Esse lapso de tempo para começar a formação do iodeto a partir do iodato foi mencionado por Waite \& Truesdale (2003) que re- portaram um tempo de retardo de 10 dias para começarem a observar a formação de iodeto durante estudos de incubação de iodato em culturas de fitoplâncton. Diferentemente do que acontece com amostras incubadas com iodeto, houve a redução de aproximadamente $50 \%$ do iodato- 131 adicionados na amostra não filtrada para iodeto-131, enquanto na amostra que sofreu processo de filtração houve conversão em torno de $25 \%$.

Com o decorrer da execução da linha de pesquisa, vislumbrou-se a importância de se buscar o desenvolvimento de experimentos envolvendo o importante compartimento geoquímico que é o sedimento. Assim, passou-se a elaborar experimentos utilizando radiotraçadores focando o estudo dos principais processos biogeoquímicos envolvidos na interface água-sedimento, buscando identificar parâmetros envolvidos no enriquecimento de espécies de iodo e outros radiotraçadores no sedimento e a liberação destas espécies para a coluna d' água, principalmente buscando estabelecer parâmetros físico-químicos (variáveis) que influenciam a biodisponibilidade desses elementos químicos (Machado et al. 2012).

\subsection{Estudo de Parâmetros Físico-Químicos na Interface Água-Sedimento}

O comportamento característico dos radionuclídeos, segundo experimento realizado por Machado et al. (2008), em outra ocasião mostraram resultados em que foram obtidos a quase total sorção dos radiotraçadores no primeiro centímetro da coluna sedimentar devido à deposição de material particulado na superfície do sedimento e a processos de difusão da água intersticial. Os resultados apresentados neste trabalho pelos testemunhos A e B seguiram esse comportamento com a remoção de 99,4 a $100 \%$ dos totais de atividades dos radionuclídeos estudados no primeiro centímetro do sedimento. Porém o testemunho $\mathrm{C}$ apresentou resultados discordantes para todos os radionuclídeos estudados, exibindo um segundo pico de atividade na profundidade de 3-4 cm. Neste testemunho, no intervalo de profundidade $0-1 \mathrm{~cm}$, as atividades foram em torno de $48,7 \%$ (Selênio-75), $55,2 \%$ (Cobalto-60) e $61,0 \%$ (Cromo-51), enquanto que no intervalo de 3-4 cm foram detectados 33,6, 24,2 e 28,6\% respectivamente (Figura 4). Nesse mesmo intervalo foi en- contrada uma menor densidade aparente seca $\left(0,4 \mathrm{~g} / \mathrm{cm}^{3}\right)$ do que os intervalos imediatamente acima e abaixo dessa fatia $\left(1,3 \mathrm{~g} / \mathrm{cm}^{3}\right)$.

Existem evidências na literatura de que a bioturbação pode promover esse transporte de elementos traço para camadas mais profundas dos sedimentos marinhos (ex. Osaki \& Sugihara 1997, Santschi et al. 1984). Embora os testemunhos estudados não apresentarem interferência visível a olho nu da bioturbação (produção de "galerias ou tocas” pela macrofauna), após a desestruturação manual da fatia, foi constatada a presença massiva de raízes da vegetação de manguezal, não observada acima dessa fatia. Essas raízes podem conferir uma menor densidade nas fatias e são as prováveis responsáveis pelo acúmulo de atividade do radiotraçador no intervalo 3-4 cm do testemunho C. Esses resultados sugerem um aumento de eficiência de retenção de elementos traço em profundidades maiores pelas raízes de manguezais (Suzuki et al. 2012).

\section{CONCLUSÕES}

O trabalho descrito permite uma visão geral da ampla aplicabilidade e do potencial de dados disponíveis utilizando técnicas radiométricas em experiências de laboratório para elucidar mecanismos de interação de radionuclídeos na interface água-sedimento. Dependendo dos requisitos

experimentais os atributos do uso de radionuclídeos para estudos de laboratório em meio ambiente de manguezal foram demonstradas através de exemplos específicos, como o Manganês-54, a compreensão dos processos cinéticos da oxidação do manganês, na coluna d'água com obtenção 
das constantes específicas da reação de oxidação. No caso do Iodo-123 e Iodo-131, os estudos revelaram o potencial dos radiotraçadores no entendimento do comportamento geoquímico do iodo na coluna d'água, quer seja por meio da individualização dos processos envolvidos na geoquímica deste elemento ou a eliminação das diferentes fontes dessas espécies que dificultam o acompanhamento e a formação de uma espécie a partir de outra. No exemplo final, os radiotraçadores mostraram ser ferramentas de análise "não destrutiva", ou seja, de amostras ambientais naturais que permitem a caracterização e elucidação do papel dos elementos de interesse ambiental nos processos biogeoquímicos em sedimentos de ambientes costeiros e marinhos. Apesar da complexidade envolvendo estudos de diferentes compartimentos biogeoquímicos, essa técnica permite quantificar diretamente o efeito cumulativo da bioturbação e da rizosfera no transporte e acumulação dos elementos traços.

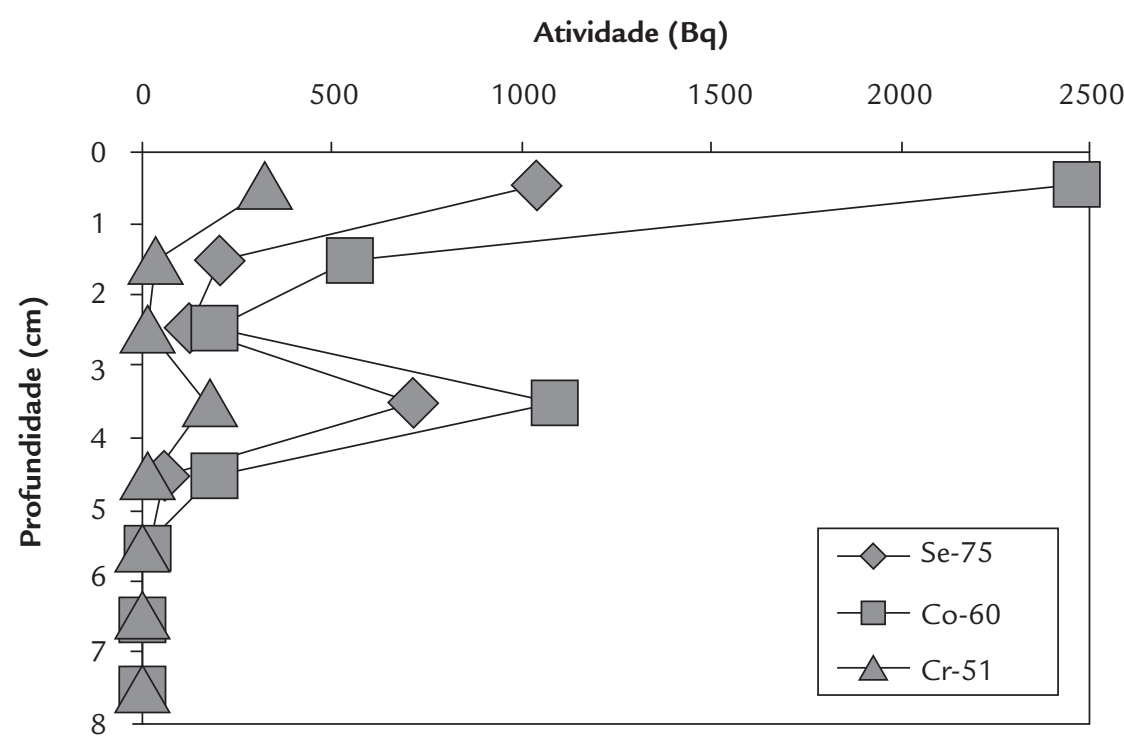

Atividade (\%)

20

40

60

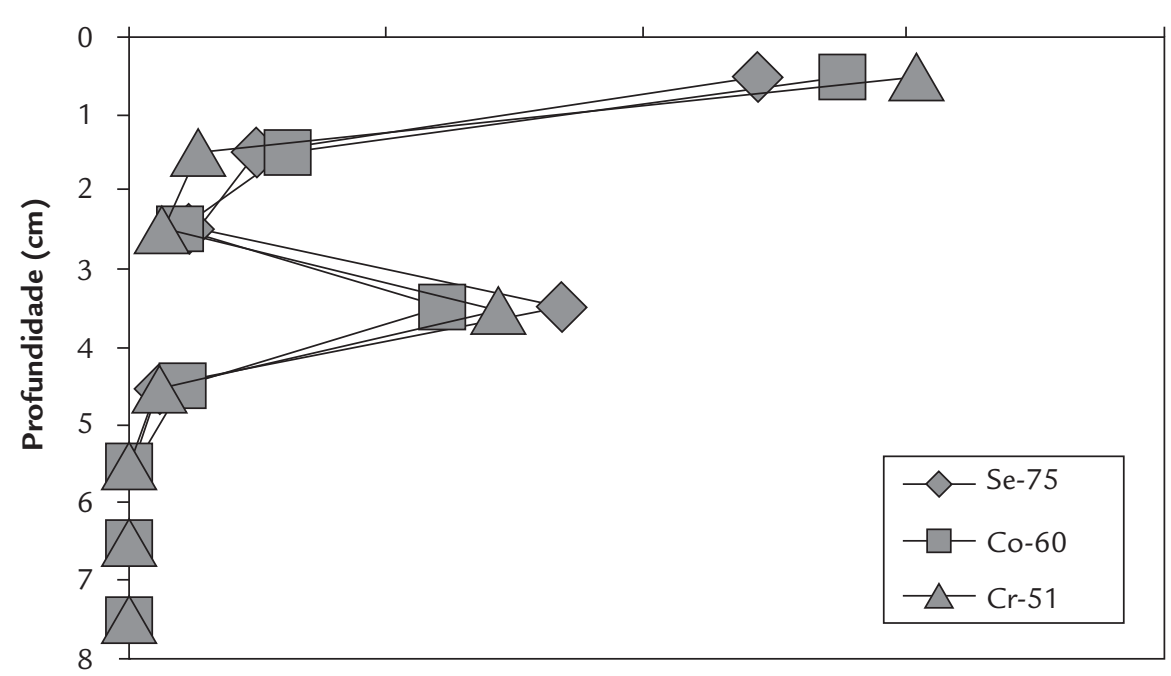




\section{AGRADECIMENTOS}

Os autores agradecem ao Dr. João A. Osso Jr. (IPEN-CNEN/SP) pela irradiação dos alvos de metais e ao Dr. Ricardo T. Lopes e à CAPES pelo auxílio na forma de bolsa PNPD.

\section{REFERÊNCIAS}

Ambe F., Ambe S., Enomoto S. 2003. Tracer Technique. In: Vértes A., Nagy S., Klenczár Z. (eds.) Handbook of Nuclear Chemistry, 3, p. 443-474.

Arthou A. 2011. Submarino de Propulsão Nuclear - Marinha do Brasil. In: I Semana da Engenharia Nuclear da UFRJ, Palestra, $n^{\circ} 9$.

Bellido A.V.B. \& Latini R.M. 2010. Fundamentos de Química Nuclear. Departamento de Físico-Química/UFF. Apostila. 117 p.

Brandão A. C. M., Rebello A. L., Wagener K. 1994. Model Experiments on the Diurnal Cycling of Iodine in Seawater. Marine Chemistry, 46:25-31.

Canesin F.P. 2000. Comportamento Cinético do Manganês numa Floresta de Manguezal - Itacuruçá, RJ. Tese Doutorado, Programa de Pós-graduação em Geociências/Geoquímica Ambiental, Universidade Federal Fluminense, $145 \mathrm{p}$.

Chopin G., Rydberg J., Liljenzin J.O. 1995. Radiochemistry and Nuclear Chemistry. Oxford, Butterworth-Heinemann Ltd., 707 pp.

Croot P.L., Heller M.I., Schlosser C., Wuttig K. 2011. Utilizing Radioisotopes for Trace Metal Speciation Measurements in Seawater. In: Nirmal Singh (Ed.). Radioisotopes - Applications in Physical Sciences. InTech, p. 247-278.

Cortez H. 2006. RJ inicia a descontaminação do terreno da Companhia Ingá Mercantil, um dos maiores passivos ambientais do estado. Disponível em: http://www.ecodebate.com.br/2009/06/05/rj-inicia-a-descontaminacaodo-terreno-da-companhia-inga-mercantil-um-dos-maiores-passivosambientais-do-estado/. Acessado em 30 maio 2011.

Edwards A. \& Truesdale V.W. 1997. Regeneration of Inorganic Iodine Species in Loch Etive, a Natural Leaky Incubator. Estuarine, Coastal and Shelf Science, 45:357-366.

Farrenkopf A.M., Dollhopf M.E., Chadhain S.N., Luther III G.W., Nealson K.H. 1997. Reduction of Iodate in seawater During Arabian Sea Shipboard Incubations and in Laboratory Culture of the Marine Bacterium Shewanella Putrefacien Strain MR-4. Marine Chemistry, 57:347-354.

Machado E.C., Bernedo A.V.B., Bernedo L.F.B. 2001. Separation of Iodine Species by Adsorption Chromatography. Journal of Radioanalytical and Nuclear Chemistry, 249(3):653-656.

Machado E.C., Bernedo A.V.B., Bernedo L.F.B., Patchineelam S.R., Machado W. 2008. Removal of Zinc from Tidal Water by Sediments of a Mangrove Ecosystem: A Radiotracer Study. Water, Air and Soil Pollution, 1:2-7.

Machado E.C., Machado W., Bellido A.V.B., Bellido L.F., Patchineelam S.R. 2012. Cesium, manganese and cobalt water-sediment transfer kinetics and diffusion into mangrove sediments inferred by radiotracer experiments. Journal of Radioanalytical and Nuclear Chemistry, 292:349-353.

Morgan J.J. \& Stumm W. 1965. Analytical chemistry of aqueous manganese. Journal American Water Works Association 57:107-119.

Morgan J.J. 1967. Chemical Equilibra and Kinectic Properties of Manganese in Natural Waters. In: Wiley and Sons (Ed.) Principles and Applications of Water Chemistry, p. 561-564.

Osaki S. \& Sugihara S. 1997. Biodiffusion of ${ }^{7} \mathrm{Be}$ and ${ }^{210} \mathrm{~Pb}$ in intertidal estuarine sediment. Journal of Environmental Radioactivity, 37:55-71.

Palágyi S., Zaduban M., Brutovsky M. 1971. Preparation and behavior of some chemical forms of radio-iodine in aqueous solution, I. Preparation of carrier-free radioiodate. Radiochemical Radioanalytical Letters, 8:79-88. 
Payne T.E., Hatje V., Itakura T., McOrist G.D., Russell R. 2004. Radionuclide applications in laboratory studies of environmental surface reactions. Journal of Environmental Radioactivity 76:237-251.

Rebello A.L., Herms F.W., Wagner K. 1990. The Cycling of Iodine as Iodate and Iodide in a Tropical Estuarine System. Marine Chemistry, 29:77-93.

Santschi P.H., Nyffeler U.P.N., O’Hara P., Buchholtz M., Broecker W.S. 1984. Radiotracer uptake on the sea floor: results from the Manop chamber deployments in the eastern Pacific. Deep-Sea Research, 31:451-468.

Sillén L. G. 1961. The Physical Chemistry of Seawater. In: Oceanography, American Association for the Advancement of Science, Publ. 67, Washington, DC.

Silva L.L., Donnici C.L., Ayala J.D., Freitas C.H., Moreira R.M., Pinto M.F. 2009. Traçadores: O uso de agentes químicos para estudos hidrológicos, ambientais, petroquímicos e biológicos. Química Nova, 32:1576-1585.

Sunda W.G. \& Huntsman S.A. 1987. Microbial oxidation of manganese in a North Caroline Estuary. Limnology Oceanography, 32(3):552-55.

Suzuki K.N., Machado E.C., Machado W., Bellido A.V.B., Bellido L.F., Osso Jr. J.A., Lopes R.T. 2012. Selenium, chromium and cobalt diffusion into mangrove sediments: radiotracer experiment evidence of coupled effects of bioturbation and rhizosphere. Water Air Soil Pollution, 223:3887-3892.

Waite T.J. \& Truesdale V.W. 2003. Iodate reduction by Isochrysis galbana is relatively insensitive to de-activation of nitrate reductase activity - are Phytoplankton really responsible for iodate reduction in seawater? Marine Chemistry, 81:137-148.

Wong G.T.F. \& Hung C. C. 2001. Speciation of Dissolved Iodine: Integrating Nitrate Uptake over Time in the Oceans. Continental Shelf Research, 21:113-128.

Wong T. F. 1995. Dissolved Iodine Across the Gulf Stream Front and in the South Atlantic Bight. Deep-Sea Research I, 42(11/12): 2005-2023. 